\title{
Lignes directrices pratiques pour les professionnels canadiens des soins oculovisuels concernant la sécheresse oculaire et la chirurgie de l'œil
}

pour le Groupe de consensus canadien sur la sécheresse oculaire

Paul M. Karpecki, O.D., FAAO, Kentucky Eye Institute, University of Pikeville School of Optometry

C. Lisa Prokopich, O.D., M. Sc., University of Waterloo,

School of Optometry

and Vision Science

Louis Racine, M.D., FRCSC, Université de Montréal, Faculté de médecine, Département d'ophtalmologie

Etty Bitton, O.D., M. Sc., FAAO, Université de Montréal, École d'optométrie, Montréal

Barbara Caffery, O.D., Ph. D., Toronto Eye Care

Paul Harasymowycz,

M.D., FRCSC,

Institut du Glaucome de Montréal

Langis Michaud,

O.D., M. Sc., FAAO,

Université de Montréal,

École d'optométrie

Victor D. Pegado, M.D., FRCSC, Dipl. ABO, University of British Columbia, Faculty of Medicine, Department of Ophthalmology and Visual Sciences

Jean-Sébastien Dufour, O.D., M. Sc., Université de Montréal, École d'optométrie

Paul Neumann, O.D., Central Saanich Optometry

Andrew Webber, O.D., Elmsdale Vision Centre

John Ashkenas10, Ph. D., SCRIPT
RÉSUMÉ :

En 2014, le Groupe de consensus canadien sur la sécheresse oculaire a publié un document intitulé Dépistage, diagnostic et prise en charge de la sécheresse oculaire : guide pratique à l'intention des optométristes canadiens. Ce guide pratique ne traitait pas des répercussions de la sécheresse oculaire chez les personnes en voie de subir une intervention chirurgicale de l'œil ou ayant récemment subi ce genre d'intervention. La sécheresse oculaire est courante dans certaines cohortes ayant subi une intervention chirurgicale, et le contexte périopératoire pose des problèmes précis; d'une part parce qu'une intervention chirurgicale peut compliquer une sécheresse oculaire préexistante et, d'autre part, parce la sécheresse oculaire symptomatique et asymptomatique expose le patient au risque d'obtenir des résultats chirurgicaux médiocres. Le groupe de consensus a élaboré cet addenda au guide pratique de 2014 pour offrir des conseils sur les soins relatifs à la sécheresse oculaire avant et après une intervention chirurgicale aux yeux.

TITRE ABRÉGÉ :

Sécheresse oculaire périopératoire

AUTEUR-RESSOURCE

Paul M. Karpecki, O.D., FAAO, Kentucky Eye Institute, 601 Perimeter Dr., bureau 100, Lexington, Kentucky, 40517, tél. : 859 278-9393, télécopieur : 859 277-3965, courriel : paul@karpecki.com

\section{MOTS CLÉS :}

sécheresse oculaire, syndrome de l'œil sec, kératoconjonctivite sèche, phacoémulsification, LASIK, qualité de la vision, douleur neuropathique oculaire 


\section{INTRODUCTION}

\section{La sécheresse oculaire et le guide pratique à l'intention des optométristes canadiens de 2014}

La sécheresse oculaire, aussi appelée kératoconjonctivite sèche, englobe un groupe de maladies inflammatoires de la surface oculaire qui, regroupées, sont omniprésentes ${ }^{1,2}$ dans l'ensemble de la population. La sécheresse oculaire est fortement répandue dans certaines sous-populations, notamment les adultes de plus de $50 \mathrm{ans}^{1}$, les femmes, les diabétiques de longue date ${ }^{3}$, et les personnes qui portent des lentilles cornéennes., ${ }^{4}{ }^{5}$ Les affections plus rares associées à une sécheresse oculaire grave sont notamment le syndrome de Sjögren (SS) ${ }^{6}$, le syndrome de StevensJohnson, la lagophtalmie nocturne, la kératite lagophtalmique et la maladie du greffon contre l'hôte., 8

La sécheresse oculaire englobe de multiples affections dans lesquelles le film lacrymal précornéen est rare, instable, inadéquatement réparti ou anormalement composé. À titre d'exemple, les larmes peuvent avoir une carence en lipides (dysfonctionnement des glandes de Meibomius; une cause courante de la sécheresse oculaire par évaporation), en eau (p. ex., le syndrome de Sjögren), ou en mucines (p. ex., en raison d'une anomalie génétique ou d'une perte de la fonction des cellules caliciformes conjonctivales). ${ }^{8}$ Chez les personnes atteintes de sécheresse oculaire, les larmes deviennent hyperosmolaires et peuvent accumuler des cytokines qui déclenchent et perpétuent l'inflammation locale. Les larmes hyperosmolaires peuvent endommager l'épithélium cornéen et conjonctival, en partie du fait qu'elles contiennent des niveaux élevés de médiateurs inflammatoires, comme la métalloprotéinase-9 de matrice enzymatique de dégradation. ${ }^{9}$

La sécheresse oculaire peut présenter toutes les combinaisons de symptômes suivants : sécheresse, fatigue, rougeur, sensation de brûlure, démangeaisons ou élancement des yeux, sensation de corps étranger, sensibilité à la lumière, filaments de mucus, irritation des paupières et croûtes sur le bord de la paupière. Les patients peuvent rapporter une vision floue ou des fluctuations de la vision, et constater une diminution de leur acuité visuelle et un dysfonctionnement des tâches visuelles. L'utilisation de colorants pour la coloration de la surface oculaire pendant les examens courants permet souvent de détecter les anomalies conjonctivales et cornéennes comme la kératite ponctuée superficielle. ${ }^{10}$ Dans les cas extrêmes, la sécheresse oculaire peut mener à l'ulcération cornéenne, à la néovascularisation, à des lésions permanentes et à une perte de vision irréversible. ${ }^{11}$

\section{ENCADRÉ LATÉRAL : DOULEUR NEUROPATHIQUE OCULAIRE}

Les patients qui souffrent de douleur oculaire neuropathique peuvent décrire leur expérience subjective en termes semblables à ceux utilisés pour décrire la sécheresse oculaire. Les mots fréquemment utilisés pour décrire la douleur découlant de ces deux affections qui peuvent toutes deux causer une sensibilité à la lumière sont notamment «brûlure », « douleur aiguë » et « démangeaisons ». Cette similitude de présentation des affections entraine souvent de la confusion et une insatisfaction par rapport au traitement, particulièrement chez ceux qui développent une douleur postopératoire persistante ou incurable. ${ }^{24,89}$

Les origines de la douleur neuropathique sont obscures, mais des dommages nerveux découlant de sources variées peuvent être une cause immédiate. ${ }^{23,34,90}$ La régénération aberrante des nerfs et plusieurs autres événements neurologiques centraux et périphériques ${ }^{22}$ ont été proposés pour expliquer la neuropathie; ils auraient entraîné la nature chronique de la douleur. ${ }^{24,89}$

La douleur aux yeux peut être désignée pour la première fois comme étant neuropathique après l'intervention chirurgicale. Toutefois, la douleur oculaire neuropathique est aussi largement répandue chez les patients qui n'ont pas d'antécédents d'intervention chirurgicale oculaire. La douleur rapportée après l'intervention chirurgicale suit parfois un modèle préexistant qui peut avoir été fautivement attribué à la sécheresse oculaire ou à d'autres causes. On doit soupçonner une origine neuropathique lorsque l'hypersensibilité oculaire, l'hyperalgésie (réponse exagérée à la douleur lors d'un stimulus nociceptif supraliminaire), ou l'allodynie (douleur en réponse à un stimulus normalement non nociceptif) ne sont pas proportionnées aux signes objectifs de la sécheresse oculaire, comme la coloration cornéenne et conjonctivale.

Cette possibilité peut être évaluée en instillant des gouttes anesthésiques, émoussant ainsi les signaux nociceptifs des neurones de la cornée. ${ }^{22,24}$ Par définition, la douleur neuropathique émane du tronc cérébral ou d'une autre région du système nerveux central. Par conséquent, bien que le test ne constitue pas un diagnostic, l'observation de la douleur qui persiste malgré l'anesthésie topique suggère qu'il s'agit de neuropathie. On a également signalé que la douleur neuropathique répondait mal aux larmes artificielles, en comparaison avec la douleur psychologique (nociceptive). ${ }^{91}$

Les comorbidités courantes sont notamment la douleur neuropathique générale (non oculaire), de même que la dépression, l'anxiété, et les troubles du sommeil; ${ }^{22,92,93}$ les patients qui signalent une douleur oculaire autrement inexpliquée devraient être interrogés au sujet de ces autres affections également. 
En 2014, le Groupe de consensus canadien sur la sécheresse oculaire a présenté le document intitulé Dépistage, diagnostic et prise en charge de la sécheresse oculaire : guide pratique à l'intention des optométristes canadiens. ${ }^{8} \mathrm{Ce}$ document d'orientation (fig. 1) offrait des principes généraux pour le diagnostic et la prise en charge de la sécheresse oculaire épisodique, chronique et récalcitrante, et pour la surveillance de l'efficacité du traitement. Selon ce guide pratique, il est essentiel que tous les professionnels des soins oculovisuels effectuent le dépistage des maladies de la surface oculaire et qu'ils prennent en charge ces maladies de manière adéquate. Comme il est exposé dans le guide pratique, la sécheresse oculaire épisodique peut parfois être prise en charge au moyen de gouttes ophtalmiques lubrifiantes, de l'hygiène des paupières et/ou de la modification des milieux de vie et de travail. En revanche, la sécheresse oculaire chronique est une maladie inflammatoire qui requiert un traitement anti-inflammatoire. ${ }^{8}$ Les options accessibles à l'heure actuelle pour prendre en charge l'inflammation de la surface oculaire sont notamment les corticostéroïdes topiques, qui sont généralement réservés à l'utilisation à court terme, de même que les acides gras essentiels et l'émulsion de cyclosporine à $0,05 \%$ dans l'huile de ricin (Restasis", Allergan Inc.; tout au long du présent addenda, la cyclosporine renvoie à cette formulation). ${ }^{8}$ Les autres produits anti-inflammatoires topiques, comme le lifitegrast à $5 \%$ et la cyclosporine à $0,1 \%$ dans une préparation cationique, pourraient bientôt être accessibles sur le marché canadien pour le traitement de la sécheresse oculaire. ${ }^{12-17}$ À ce jour, il n'y a eu aucun rapport sur l'usage périopératoire de ces autres produits.

Figure 1. Diagnostic et prise en charge de la sécheresse oculaire, selon le guide pratique à l'intention des optométristes canadiens de $2014^{8}$

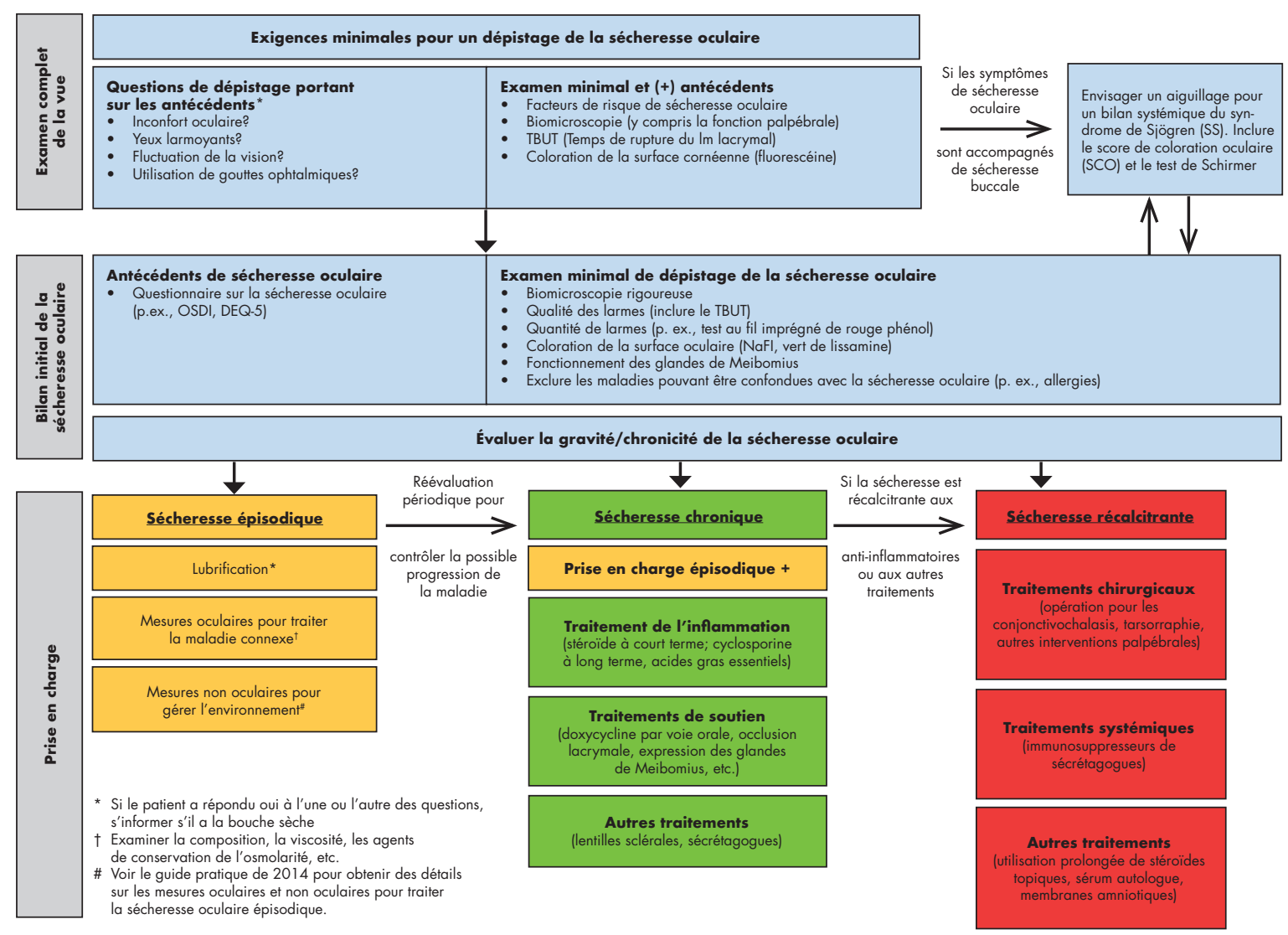

\section{Portée et objectifs de cet addenda}

Le guide pratique de 2014 ne traitait pas de la prise en charge de la sécheresse oculaire chez les personnes ayant recours à une intervention chirurgicale oculaire. Ce sujet est important étant donné les diverses façons dont la sécheresse oculaire non maîtrisée peut devenir un problème pour les patients ayant recours à une opération de la cataracte ou à une intervention de chirurgie réfractive. Par ailleurs, les interventions chirurgicales oculaires en tous genres (tableau 1) peuvent causer l'inflammation de la surface oculaire, laquelle résulte d'un traumatisme direct à la cornée, d'une légère toxicité du microscope chirurgical, de l'évaporation du film lacrymal pendant l'intervention chirurgicale, ou d'une irritation causée par les anesthésiques topiques, les solutions antiseptiques de surface et les gouttes ophtalmiques contenant des agents de conservation. ${ }^{18-21}$ Par conséquent, une intervention chirurgicale peut précipiter le retour de la sécheresse oculaire ou exacerber l'affection chez les patients ayant une sécheresse oculaire symptomatique ou asymptomatique préexistante. 
La sécheresse oculaire et la douleur postopératoire qui se manifestent pendant la convalescence sont habituellement temporaires, mais peuvent persister chez certaines personnes. Une douleur chronique sans maladie de la surface oculaire ( « douleur sans tache »)22 peut aussi se manifester, et peut être présente avant ou après l'intervention chirurgicale; ce genre de douleur oculaire neuropathique, résultant de lésions dans le système nerveux somatosensoriel, ${ }^{23}$ ne se distingue pas aisément de la sécheresse oculaire. Cette confusion pose divers problèmes cliniques qui peuvent être frustrants pour les patients et les fournisseurs de soins (voir l'encadré latéral sur la douleur neuropathique oculaire).22,24

Comme il en sera question plus loin, une maladie de la surface oculaire préexistante complique la biométrie préopératoire, la kératométrie, la topographie et la réfraction, augmentant ainsi le risque pour le patient d'une correction visuelle insatisfaisante et d'une régression réfractive. La sécheresse oculaire peut diminuer la qualité de la vision après une intervention chirurgicale, ce qui peut engendrer de l'inconfort et de l'insatisfaction, même chez les patients dont l'acuité visuelle a été corrigée efficacement. En revanche, les mesures qui rétablissent le film lacrymal, réduisent l'osmolarité des larmes, ou suppriment l'inflammation cornéenne et conjonctive peuvent améliorer les symptômes postopératoires et les résultats visuels. Par conséquent, il y a plusieurs raisons impérieuses de prendre en charge la sécheresse oculaire, autant avant qu'après une intervention chirurgicale. Néanmoins, chez les candidats à l'intervention chirurgicale et chez d'autres personnes, la maladie de la surface oculaire passe souvent inaperçue et n'est pas traitée. ${ }^{8,25} \mathrm{Il}$ est essentiel que les professionnels des soins oculovisuels reconnaissent les signes et les symptômes de la sécheresse oculaire chez les candidats à une intervention chirurgicale pour commencer rapidement le traitement et, dans la mesure du possible, pour renverser l’inflammation de la surface oculaire avant de procéder à l'intervention chirurgicale.

Tableau 1. Interventions chirurgicales de l'œil qui peuvent nécessiter un diagnostic et une prise en charge de la sécheresse oculaire (SO)

\begin{tabular}{|c|c|c|c|c|}
\hline Type d'intervention chirurgicale & $\begin{array}{l}\text { Est-ce que } \\
\text { l'intervention provoque } \\
\text { ou complique la SO? }\end{array}$ & $\begin{array}{l}\text { Est-ce que la } \\
\text { SO complique } \\
\text { l'intervention? }\end{array}$ & $\begin{array}{l}\text { Est-ce que la SO } \\
\text { est très courante } \\
\text { dans la cohorte } \\
\text { chirurgicale? }\end{array}$ & Références \\
\hline \multicolumn{5}{|l|}{ Réfractive } \\
\hline $\begin{array}{l}\text { LASIK (kératomileusie in situ au } \\
\text { laser) }\end{array}$ & Oui & Oui & Oui & $\begin{array}{l}\text { Le présent } \\
\text { article }\end{array}$ \\
\hline PRK (photokératectomie réfractive) & Oui & Oui & Oui & 110 \\
\hline \multicolumn{5}{|l|}{ Cataracte } \\
\hline Phacoémulsification & Oui & Oui & Oui & $\begin{array}{l}\text { Le présent } \\
\text { article }\end{array}$ \\
\hline \multicolumn{5}{|l|}{ Cornée/conjonctive } \\
\hline Kératoplastie pénétrante & Oui & Oui & Oui & $111-113$ \\
\hline Kératoplastie endothéliale & Oui & Oui & Oui & $111-114$ \\
\hline Excision d'un ptérygion & Oui & Non & Oui & 115 \\
\hline $\begin{array}{l}\text { Enlèvement d'un } \\
\text { conjonctivochalasis }\end{array}$ & Non & Oui & Oui & $116-119$ \\
\hline \multicolumn{5}{|l|}{ Vitréo rétinienne } \\
\hline Vitrectomie & Oui & $?$ & Oui & 120,121 \\
\hline \multicolumn{5}{|l|}{ Paupières } \\
\hline Blépharoplastie & Oui & Non & Oui & $122-124$ \\
\hline Réparation du ptosis & Oui & $?$ & Non & 122 \\
\hline \multicolumn{5}{|l|}{ Glaucome } \\
\hline Trabéculectomie & Oui & Oui & Oui & 125,126 \\
\hline \multicolumn{5}{|l|}{ Autres } \\
\hline Correction du strabisme & Oui & Non & Non & 127 \\
\hline
\end{tabular}


Par conséquent, nous avons rédigé cet addenda au document Dépistage, diagnostic et prise en charge de la sécheresse oculaire : guide pratique à l'intention des optométristes canadiens de 2014 précisément pour assurer la prise en charge périopératoire de la sécheresse oculaire. L'accent est mis ici sur l'opération de la cataracte, principalement la phacoémulsification avec implantation d'une lentille intraoculaire et sur les procédures réfractives comme la kératomileusie in situ au laser (LASIK). On traite de la sécheresse oculaire comorbide chez les personnes atteintes de glaucome dans l'encadré latéral sur la sécheresse oculaire chez les personnes atteintes de glaucome.

\section{ENCADRÉ LATÉRAL: SÉCHERESSE OCULAIRE ASSOCIÉE AU GLAUCOME}

La sécheresse oculaire est une comorbidité courante du glaucome, qui se présente chez $15 \%$ à $59 \%$ des patients atteints de glaucome. ${ }^{94-99}$ Cette association peut s'expliquer au moins en partie par la polypharmacie des gouttes ophtalmiques médicamenteuses, et plus précisément par la présence et la durée de l'exposition aux irritants qui se trouvent dans les produits médicamenteux et non médicamenteux, notamment les ingrédients pharmaceutiques actifs et les agents de conservation comme le chlorure de benzalkonium (BAK). ${ }^{99,100}$

L’inflammation résultant du BAK peut être accentuée par la présence de larmes hyperosmolaires, ${ }^{101}$ qui sont courantes chez les personnes atteintes de sécheresse oculaire. En outre, l'utilisation chronique de produits contenant du BAK peut entraîner une augmentation de l'osmolarité du film lacrymal, et la sécheresse oculaire peut compliquer l'intervention chirurgicale en ophtalmologie, y compris les interventions de traitement du glaucome. ${ }^{94}$ Chez les patients qui utilisent des produits contenant du BAK, un traitement préopératoire de stéroïdes topiques peut diminuer l'inflammation conjonctivale et augmenter le taux de succès des trabéculectomies; bien entendu, il est essentiel de surveiller la pression intraoculaire lors de l'utilisation de stéroïdes. ${ }^{102,103}$

Les larmes artificielles sans agent de conservation sont associées à la réduction des symptômes de sécheresse oculaire ${ }^{97}$ et devraient être utilisées de préférence aux larmes artificielles avec agents de conservation. ${ }^{8}$ Toutefois, il est rarement possible pour les patients atteints de glaucome d'éviter l'utilisation de médicaments topiques irritants, particulièrement lorsque la maladie évolue et qu'ils doivent abandonner la monothérapie topique. ${ }^{100,104,105}$ Pour cette raison, on peut envisager un traitement anti-inflammatoire pour améliorer la sécheresse oculaire chez les patients qui devront s'exposer aux médicaments topiques contre le glaucome pendant une longue période. ${ }^{106} \mathrm{Si}$ l'on présume généralement que la maîtrise du glaucome est l'objectif principal lorsque ces deux affections coexistent, de récentes constatations suggèrent que l'optimisation de la surface chez les patients atteints d'un glaucome concomitant est compatible avec la réduction de la pression intraoculaire. ${ }^{107,108}$

La trabéculectomie et d'autres interventions chirurgicales pour traiter le glaucome exacerbent temporairement la sécheresse oculaire, mais offrent la possibilité d'une maîtrise à long terme de la pression intraoculaire en plus dépendance fortement réduite des médicaments topiques contre le glaucome. En effet, $40 \%$ des patients n'ont plus besoin de médicaments topiques contre le glaucome pendant une période pouvant aller jusqu'à trois ans après la trabéculectomie. ${ }^{109}$ Depuis l'émergence d'interventions chirurgicales moins invasives, il peut être possible de permettre une meilleure maîtrise de la pression intraoculaire plus tôt dans la progression du glaucome, réduisant ainsi l'exposition chronique aux médicaments topiques irritants. Ce qu'on appelle les interventions chirurgicales micro-invasives du glaucome (ICMIG) sont des interventions ab interno, ce qui signifie qu'elles sont effectuées à partir de l'intérieur de l'œil et qu'elles causent un trauma minimal ou nul à la conjonctive. Les ICMIG sont considérées comme présentant un risque assez faible pour que leur utilisation puisse être justifiée même chez les personnes atteintes de glaucome léger à modéré96 Bien qu'on manque encore de données probantes directes, les ICMIG devraient par conséquent présenter moins de risque de provoquer la sécheresse oculaire en comparaison des interventions chirurgicales classiques. 
Les approches générales de prise en charge de la sécheresse oculaire décrites dans le guide pratique à l'intention des optométristes canadiens s'appliquent aux patients qui ont besoin d'une intervention chirurgicale. C'est pour cette raison que le présent addenda est centré sur des questions propres aux soins périopératoires de la sécheresse oculaire, notamment les suivantes :

Quels éléments probants désignent la sécheresse oculaire non maîtrisée dans le cas de résultats postopératoires indésirables?

Quelle devrait être l'incidence de la présence de sécheresse oculaire dans la décision concernant le choix du moment ou la pertinence d'une intervention?

De quelle façon devrait-on prendre en charge la sécheresse oculaire avant et après une intervention?

Comment les optométristes et les ophtalmologistes devraient-ils prendre en charge conjointement

la sécheresse oculaire chez les personnes retenues pour subir une intervention chirurgicale oculaire?

SÉCHERESSE OCULAIRE, FONCTIONNEMENT VISUEL ET BIOMÉTRIE OCULAIRE

Le film lacrymal précornéen, qui est la première surface réfractive de l'œil, fonctionne de façon optimale quand il est lisse comme un miroir. Le film lacrymal est entretenu par des mécanismes neuroendocriniens qui régulent la fonction sécrétoire et la fréquence de clignement des yeux en réponse aux stress environnementaux changeants. ${ }^{10,26,27}$ Un film lacrymal sain est suffisamment épais, uniforme, et équilibré avec les composants appropriés pour protéger la surface oculaire des agressions et pour éviter les aberrations optiques entre les clignements. ${ }^{28-31}$

Des anomalies du film lacrymal, qui affectent soit la quantité ou la composition des larmes, peuvent mener à des maladies de la surface oculaire dues à la déficience aqueuse ou à l'évaporation. Un trauma chirurgical compromet la régulation du film lacrymal, au moins temporairement. À titre d'exemple, la perte de sensibilité tactile aux sites dénervés chirurgicalement dans la cornée nuit au larmoiement de base et par réflexe, en plus de réduire la fréquence du clignement des yeux, ce qui compromet le film lacrymal et la surface oculaire, pendant que les nerfs endommagés se reforment. ${ }^{27,32-34}$

La sécheresse oculaire affecte la qualité de la vision sans nécessairement détériorer l'acuité visuelle.,29,35 À titre d'exemple, la sécheresse oculaire est associée à un éblouissement, à un dérèglement de la sensibilité différentielle et à des symptômes d'aberrations d'ordre supérieur; les patients ont une vision changeante durant l'intervalle entre les clignements successifs, en raison d'un film lacrymal instable qui n'est pas uniforme. ${ }^{27}$ La qualité de la vision est un facteur prédictif important du fonctionnement quotidien, particulièrement pour les tâches qui sont très exigeantes envers le système visuel comme la lecture ou la conduite automobile. ${ }^{36,37}$

Chez les patients qui subissent une intervention chirurgicale oculaire, les troubles de la vue liés à la sécheresse oculaire peuvent accroître le risque d'insatisfaction postopératoire du patient. La planification chirurgicale pour l'intervention de chirurgie réfractive et pour l'opération de la cataracte avec implantation d'une lentille intraoculaire nécessite une kératométrie et/ou une topographie exactes. Une sécheresse oculaire non maîtrisée peut altérer la forme de la cornée et ${ }^{38}$ réduire ainsi l'exactitude et la précision des constatations biométriques. L'hyperosmolarité des larmes est associée à une plus grande dispersion statistique des mesures kératométriques. L'irrégularité et l'instabilité de la surface oculaire réduisent la précision des calculs préopératoires relatifs à la lentille intraoculaire et à la réfraction, ce qui accroît le risque de réfraction sous-optimale après l'intervention chirurgicale, particulièrement lors de l'utilisation d'implants toriques ou multifocaux. ${ }^{39}$ Par ailleurs, même chez les personnes qui obtiennent une bonne acuité visuelle postopératoire, la sécheresse oculaire peut réduire la qualité de la vision au cours d'une période de quelques semaines ou de quelques mois, et parfois plus longtemps. ${ }^{40}$

Le tableau 2 résume les divers objectifs du traitement de la sécheresse oculaire dans l'ensemble de la population et chez les candidats à l'intervention de chirurgie oculaire. 


\begin{tabular}{|l|l|}
\hline $\begin{array}{l}\text { Pour l'ensemble des personnes atteintes de } \\
\text { sécheresse oculaire }\end{array}$ & $\begin{array}{l}\text { Pour les personnes atteintes de sécheresse oculaire } \\
\text { qui subissent une intervention chirurgicale }\end{array}$ \\
\hline $\begin{array}{l}\text { - Améliorer l'inconfort et la fatigue oculaires } \\
\text { - Prévenir l'érosion cornéenne et les anomalies de } \\
\text { surface associées à la maladie de la surface oculaire } \\
\text { et à l'hyperosmolarité des larmes }\end{array}$ & $\begin{array}{l}\text { Obtenir des mesures réfractives de biométrie/kératométrie } \\
\text { plus exactes et plus précises pour calculer avec plus } \\
\text { d'assurance les paramètres de la puissance des lentilles } \\
\text { intraoculaires ou de la surface LASIK }\end{array}$ \\
$\begin{array}{l}\text { Prévenir les aberrations optiques qui réduisent } \\
\text { la qualité de la vue (p. ex., vision floue, éblouissement, } \\
\text { perte de sensibilité aux contrastes) }{ }^{30,35}\end{array}$ & $\begin{array}{l}\text { Améliorer l'acuité visuelle et la qualité de la vision } \\
\text { postopératoire } \\
\text { Prévenir ou réduire au minimum la sécheresse oculaire } \\
\text { postopératoire }{ }^{40}\end{array}$ \\
$\begin{array}{l}\text { Améliorer la performance et la facilité de la demande } \\
\left.\text { des tâches visuelles (p. ex., la lecture }{ }^{36} \text { et la conduite }{ }^{37}\right)\end{array}$ & \\
\hline
\end{tabular}

\section{Le besoin croissant de soins concertés pour la sécheresse oculaire}

Plusieurs tendances démographiques et sociales ont accru l'urgence et le fardeau de la prise en charge de la sécheresse oculaire. Premièrement, la sécheresse oculaire et les interventions chirurgicales aux yeux sont de plus en plus courantes à mesure que la population vieillit. Deuxièmement, la dépendance accrue à l'égard des dispositifs électroniques et leur utilisation croissante peuvent causer ou exacerber la sécheresse oculaire en réduisant la fréquence de clignement des yeux chez l'utilisateur. ${ }^{41,42}$ Les personnes qui utilisent ces dispositifs doivent avoir un haut niveau de fonctionnement visuel et peuvent souhaiter avoir recours à une intervention chirurgicale précisément pour améliorer la qualité de leur vision. Troisièmement, la technologie d'ophtalmologie elle-même a évolué avec l'introduction des techniques de mesure qui offrent une précision sans précédent en biométrie oculaire, mais qui dépendent d'un film sain pour une utilisation optimale. De ma même façon, les lentilles intraoculaires multifocales et toriques offrent la possibilité de corriger la vision de près et de loin, mais elles semblent plus sensibles aux aberrations visuelles et aux erreurs de biométrie que la génération précédente de lentilles intraoculaires. ${ }^{43}$ Cette différence est inhérente à la technologie des lentilles intraoculaires, mais l'insatisfaction résulte aussi en partie d'attentes plus élevées, notamment du désir d'un fonctionnement visuel optimal et d'une dépendance minimale des lunettes pour la vision éloignée ou rapprochée.

Pour toutes ces raisons, le besoin croissant de soins diligents pour la sécheresse oculaire avant et après l'intervention chirurgicale exige de plus en plus d'heures des fournisseurs de soins. Des approches efficaces, dont la prise en charge concertée de la sécheresse oculaire par les optométristes et les ophtalmologistes, seront nécessaires pour répondre à ces demandes.

\section{OPÉRATION DE LA CATARACTE}

Si nous extrapolons les données de 2014 provenant d'une seule province, environ 450000 interventions de phacoémulsification peuvent être exécutées chaque année à l'échelle du Canada, ce qui fait des cataractes la raison la plus fréquente d'avoir recours à une intervention de chirurgie oculaire. ${ }^{44,45} \mathrm{La}$ demande pour ce genre d'intervention devrait plus que doubler d'ici $2036 .{ }^{46}$

La norme actuelle de soins pour l'extraction de cataracte est la phacoémulsification suivie d'une implantation de lentille intraoculaire, qui peut être soit monofocale ou « Premium ». Ce dernier type de lentille intraoculaire comprend une variété de modèles qui permettent la correction de la vision éloignée, rapprochée et intermédiaire, ce qui permet généralement une moins grande dépendance à l'égard des lunettes ou des lentilles cornéennes. Selon le modèle, les lentilles intraoculaires Premium pourraient être sujettes à l'éblouissement et aux halos, il est donc important de réduire au minimum les autres perturbations visuelles chez ces patients, notamment les aberrations liées à la sécheresse oculaire. ${ }^{4748}$ Fait intéressant, les cataractes elles-mêmes peuvent provoquer des aberrations d'ordre supérieur, ${ }^{49,50}$ et cet effet sur la qualité de la vision est compliqué par une sécheresse oculaire mal maîtrisée. ${ }^{29,30}$

Une sécheresse oculaire préexistante est courante chez les personnes qui ont des cataractes, puisque certains facteurs de risque (nommément le vieillissement, le diabète ${ }^{3}$, le fait d'être une femme ${ }^{51}$ ) prédisposent les patients à ces deux affections..$^{52}$ Toutefois, la sécheresse oculaire passe souvent inaperçue et n'est donc pas traitée chez les patients qui subissent une opération de la cataracte. Trattler et coll. ont déclaré que, sur 136 patients américains qui subissaient une opération de la cataracte, si seulement $22 \%$ des patients avaient un diagnostic préalable de 
sécheresse oculaire, une plus grande proportion de patients montraient des signes objectifs de maladie de la surface (temps de rupture du film lacrymal [TBUT] $\leq 5$ secondes, $63 \%$; coloration cornéenne, $77 \%$ ), ce qui suggérait un sous-diagnostic généralisé. ${ }^{25}$ Autre fait intéressant, les symptômes subjectifs de la sécheresse oculaire étaient moins courants, puisque seulement $31 \%$ des répondants ont déclaré une sensation de brûlure, et $41 \%$ ont déclaré sentir la présence d'un corps étranger. Ces constatations sont conformes aux rapports antérieurs qui suggèrent que l'autodéclaration des patients n'est pas un outil de dépistage fiable pour la sécheresse oculaire. ${ }^{41,53,54}$

La sécheresse oculaire non maîtrisée limite l'exactitude de la biométrie préopératoire et entraîne des erreurs sur le plan de la puissance ou du positionnement des lentilles intraoculaires. ${ }^{41,54}$ Cet effet a été clairement démontré au moyen de données prélevées lors de lectures répétées chez les patients qui se présentent pour une opération de la cataracte. Epitropoulos et coll. ont rapporté que la différence moyenne entre deux lectures kératométriques successives était de 0,28 D chez les personnes qui ont des larmes hyperosmolaires ( $\mathrm{n}=100$ yeux) par rapport à $0,13 \mathrm{D}$ dans le groupe de contrôle ( $\mathrm{n}=50$ yeux). Les différences de puissance calculées des lentilles intraoculaires atteignaient jusqu'à 5,5 D dans le groupe de 100 yeux ayant des larmes hyperosmolaires, et la fréquence d'une différence de puissance $\geq 0,5 \mathrm{D}$ dans les lentilles intraoculaires était considérablement plus élevée pour les larmes hyperosmolaires que pour les larmes normales $(\mathrm{p}=0,02)$. Par ailleurs, $17 \%$ des yeux qui ont des larmes hyperosmolaires, mais seulement $2 \%$ des yeux qui ont des larmes normales affichaient une différence $\geq 1,0 \mathrm{D}(\mathrm{p}=0,01)$ du vecteur d'astigmatisme entre les lectures. ${ }^{39}$

\section{Apparition des symptômes de la sécheresse oculaire après une opération de la cataracte}

L'opération de la cataracte perturbe la surface oculaire et provoque l'inflammation intraoculaire et de la surface oculaire. Par ailleurs, l'intervention chirurgicale endommage les neurones sensoriels et les autres neurones, et l'énervation qui en résulte réduit la sensibilité tactile et autres sensibilités dans la cornée..$^{40}$ Le retour des symptômes de sécheresse oculaire est courant après la phacoémulsification, ${ }^{10,27}$ mais ces symptômes sont habituellement temporaires. L'hypoesthésie cornéenne, l'instabilité du film lacrymal et d'autres indicateurs de la sécheresse oculaire se résorbent souvent dans une période de trois mois, laquelle coïncide probablement avec le début de la régénération axonale. ${ }^{18,19}$ La sensibilité cornéenne revient graduellement à des niveaux quasi préopératoires sur une période d'un an. ${ }^{40}$ Toutefois, chez un petit sous-ensemble de patients, les symptômes de la sécheresse oculaire persistent indéfiniment. ${ }^{20,55}$ À titre d'exemple, les personnes atteintes de diabète présentent un risque accru de sécheresse oculaire postopératoire grave et chronique. ${ }^{56}$

Les traitements topiques doivent être appliqués de manière uniforme après l'intervention chirurgicale pour limiter l'étendue ou la durée du retour de la sécheresse oculaire. ${ }^{52}$ Lorsqu'ils sont utilisés de concert avec les stéroïdes topiques, on a rapporté que les lubrifiants amélioraient les symptômes de sécheresse oculaire et le fonctionnement visuel, par rapport aux soins topiques postopératoires habituels administrés seuls. ${ }^{57,58}$ Jee et coll. ont comparé directement les effets des gouttes ophtalmiques lubrifiantes et contenant des stéroïdes, sans agents de conservation et avec agents de conservation, après l'opération de la cataracte, chez 80 patients (80 yeux) atteints de sécheresse oculaire avant l'opération. Dans le cadre de cette étude prospective en mode libre, les patients ont reçu les produits sans agents de conservation ou les produits avec agents de conservation quatre fois par jour pendant un mois, et deux fois par jour par la suite. Après le premier mois suivant l'intervention chirurgicale, les sujets qui avaient reçu le traitement topique sans agents de conservation ont signalé des symptômes moins graves en comparaison des symptômes de ceux qui avaient reçu le traitement avec agents de conservation $(p<0,05)$. Après le deuxième mois, les mesures objectives de la sécheresse oculaire (coloration, stabilité du film lacrymal, marqueurs inflammatoires et cellules à gobelet de la conjonctive) s'étaient considérablement améliorées grâce au traitement sans agents de conservation. ${ }^{59}$

La lubrification à elle seule pourrait être insuffisante pour soulager l'inflammation qui entraîne la sécheresse oculaire chronique. ${ }^{60-62}$ Cela est ressorti le plus clairement dans une étude multicentrique randomisée auprès de 233 adultes chinois atteints de sécheresse oculaire modérée à grave au départ. Les patients ont été affectés par randomisation à l'un des deux groupes de l'étude et devaient faire l'application deux fois par jour des produits suivants : les patients de l'un des deux groupes ont appliqué la cyclosporine 0,05\% et les patients de l'autre groupe ont utilisé l'émulsion qui servait d'excipient; aucun autre traitement n'était autorisé à l'exception des larmes artificielles. Si les deux groupes ont constaté une importante amélioration des symptômes par rapport aux symptômes initiaux, une amélioration considérablement plus importante a été observée sur le plan de la coloration de la cornée après 4 et 8 semaines ( $p=0,025$ et 0,05 , respectivement) et dans le score de Schirmer après 4 semaines $(p=0,035)$ dans le groupe de la cyclosporine par rapport au groupe de l'excipient. ${ }^{60}$ Bien qu'aucune étude contrôlée par rapport à l'excipient de ce genre n'ait été rapportée dans un contexte chirurgical, une étude prospective contrôlée de manière controlatérale 
a comparé la cyclosporine topique et la solution physiologique chez 32 patients subissant une phacoémulsification bilatérale. Chez ces patients, le traitement à la cyclosporine topique a considérablement amélioré la stabilité du film lacrymal et d'autres mesures de la sécheresse oculaire, par rapport à la solution physiologique utilisée seule. Si l'intensité de la sécheresse oculaire déclarée par les patients s'est améliorée après un premier mois de traitement, les avantages cliniques sont devenus statistiquement significatifs après deux mois de traitement à la cyclosporine. ${ }^{63}$

\section{Exacerbation de la sécheresse oculaire après l'opération de la cataracte}

Chez les patients atteints de sécheresse oculaire chronique préexistante, un traitement anti-inflammatoire peut améliorer l'acuité postopératoire et le fonctionnement visuel.47,64 Dans le cadre d'une petite étude prospective contrôlée de manière controlatérale, randomisée et en double aveugle, Donnenfeld et coll. ont comparé les gouttes ophtalmiques lubrifiantes ( $0,4 \%$ de polyéthylèneglycol 400; 0,3 \% de propylèneglycol) à la cyclosporine topique chez les patients qui se soumettent à une phacoémulsification bilatérale avec implantation de lentilles intraoculaires multifocales. Parmi les 14 participants à l'étude, seulement trois avaient un diagnostic de sécheresse oculaire. Toutefois, étant donné que le TBUT moyen au départ était faible (environ 6 secondes), d'autres participants avaient peutêtre une sécheresse oculaire non diagnostiquée ou marginale. Le traitement a commencé un mois avant l'opération et a été maintenu après l'intervention. Deux mois après l'opération (c.àd. après trois mois de traitement topique), l'acuité visuelle non corrigée était considérablement meilleure chez les participants dont les yeux avaient été traités à la cyclosporine que chez les participants ayant reçu le traitement aux lubrifiants ( $\mathrm{p}=0.005)$. La sensibilité aux contrastes a aussi été améliorée par le traitement à la cyclosporine, et il y avait une tendance numérique vers une plus grande stabilité lacrymale après deux mois. Par ailleurs, la coloration cornéenne, qui s'est aggravée par rapport à la coloration initiale dans les yeux traités au lubrifiant, s'est améliorée considérablement dans le groupe des yeux traités à la cyclosporine ( $\mathrm{p}=0,034$ pour la différence entre les groupes au deuxième mois). ${ }^{47}$

D'autres études ont exploré l'utilisation postopératoire de la cyclosporine topique après l'opération de la cataracte. ${ }^{63-65} \mathrm{Il}$ a été suggéré que certains effets de traitement sont observables dans les semaines suivant la phacoémulsification ${ }^{65}$ et d'autres interventions chirurgicales, ${ }^{66}$ mais ces allégations sont difficiles à évaluer, étant donné que les avantages établis de la cyclosporine se produisent avec une utilisation de plus longue durée ( $\geq 3$ mois).8,54,61

\section{CHIRURGIE RÉFRACTIVE}

L'intervention LASIK et d'autres interventions photoréfractives connexes sont largement utilisées pour améliorer l'acuité visuelle non corrigée. Ces interventions produisent généralement des résultats favorables et une forte satisfaction chez les patients. ${ }^{67,68}$ Toutefois, la sécheresse oculaire postopératoire est une complication possible de ces interventions qui peut causer de l'inconfort, une baisse de la vue et une insatisfaction générale. Comme dans le cas de l'opération de la cataracte (plus haut), la sécheresse oculaire après l'intervention LASIK est associée aux effets du trauma chirurgical sur la surface oculaire, notamment la perte de sensibilité dans la cornée ${ }^{40}$ et l'inhibition du clignement des yeux et du larmoiement réflexe et de base. ${ }^{69}$ La sécheresse oculaire préexistante est probablement courante et sous-diagnostiquée, étant donné que l'intolérance aux lentilles cornéennes, qui a été associée à la sécheresse oculaire, est un facteur courant qui incite les patients à demander l'intervention LASIK. ${ }^{67}$

\section{Le facteur de la sécheresse oculaire dans la sélection des patients}

Puisque les interventions chirurgicales réfractives sont des opérations chirurgicales non urgentes, seules les personnes qui ont ou qui peuvent avoir une santé adéquate de la surface oculaire sont considérés comme de bons candidats. ${ }^{2,32,70,71}$ La sécheresse oculaire grave causée par le syndrome de Sjögren ou d'autres problèmes immunitaires est habituellement considérée comme une contre-indication pour le traitement de chirurgie réfractive. Toutefois, certains rapports suggèrent que même ces personnes pourraient subir l'intervention LASIK si l'affection est prise en charge avec succès avant l'intervention. ${ }^{68,72}$ Pour ce faire, la sécheresse oculaire préexistante doit être prise en charge de manière progressive (fig. 1), à l'aide des traitements décrits dans le guide pratique de $2014 .^{8}$ Fait à remarquer, une étude rétrospective a révélé que le traitement à la cyclosporine pendant une moyenne de 3,2 mois (plage de 1 à 12 mois) permettait aux personnes atteintes de sécheresse oculaire légère d'avoir recours à la chirurgie réfractive. ${ }^{73}$

Les autres interventions chirurgicales photoréfractives, y compris les interventions sans découpe de volet cornéen comme la technique SMILE (small-incision lenticule extraction), semblent causer moins de lésions aux nerfs et une sécheresse oculaire moins grave que la technique LASIK. ${ }^{74,75} \mathrm{Il}$ n'a pas encore été déterminé si ces nouvelles interventions sont préférables pour les patients à risque élevé de sécheresse oculaire. ${ }^{69,72}$ 


\section{Sécheresse oculaire transitoire et sécheresse oculaire chronique après l'intervention LASIK}

Après l'intervention LASIK, l'instabilité du film lacrymal et d'autres signes et symptômes de sécheresse oculaire se résorbent habituellement de façon spontanée, mais une sécheresse oculaire postopératoire chronique, qui persiste pendant 6 à 12 mois après l'intervention LASIK, a été signalée chez $0,8 \%$ à $20 \%$ des patients. ${ }^{70,76}$ Le risque de sécheresse oculaire chronique après l'intervention LASIK augmente avec l'âge et est plus élevé chez les femmes que chez les hommes. ${ }^{71,73}$ On a également suggéré que les interventions LASIK pour l'hypermétropie comportent un plus grand risque de sécheresse oculaire chronique que les interventions LASIK pour la myopie. ${ }^{73,7778}$ Après l'intervention LASIK pour l'hypermétropie, les patients atteints de sécheresse oculaire préexistante et ceux qui ont développé une sécheresse oculaire chronique semblent présenter un risque plus élevé de développer une régression réfractive. ${ }^{70,77}$

Par ailleurs, la sécheresse oculaire légère préexistante ou infraclinique semble être un facteur de risque pour la sécheresse oculaire chronique après l'invention LASIK. ${ }^{32}$ Une étude prospective a suivi 139 yeux soumis à l'intervention LASIK. Les sujets étaient exclus de l'étude s'ils avaient un diagnostic définitif de sécheresse oculaire, mais ils pouvaient être inclus dans l'étude s'ils n'avaient que des symptômes isolés comme une coloration légère ou modérée de la cornée ou un TBUT inférieur à 5 secondes. Dans cette population, une coloration cornéenne préopératoire et une faible production de larmes étaient associées avec une sécheresse oculaire persistante un an après l'intervention LASIK. ${ }^{76}$ De la même façon, les patients dont la stabilité du film lacrymal était réduite au départ étaient considérablement plus à risque de développer des anomalies de surface au moins au cours des six derniers mois après l'intervention LASIK. ${ }^{79}$

L'utilisation de la cyclosporine topique suivant l'intervention LASIK a été étudiée comme supplément possible aux soins normalisés après l'intervention pour les patients qui n'ont pas de diagnostic préalable de sécheresse oculaire. Peyman et coll. ont signalé que, dans une série de 22 sujets traités bilatéralement, les yeux qui ont reçu de la cyclosporine tous les jours étaient considérablement plus susceptibles de recouvrer la sensibilité tactile dans une période de trois mois suivant l'intervention LASIK par rapport aux yeux du groupe de contrôle $(\mathrm{p} \leq 0,011) .{ }^{80}$ Le délai de cette réaction est conforme aux éléments probants qui indiquent que les avantages cliniques de la cyclosporine deviennent plus significatifs après un traitement de 3 mois ou plus. ${ }^{8,61,66}$

L'acuité visuelle après l'intervention LASIK est moins satisfaisante chez les patients qui développent une sécheresse chronique que chez les patients qui n'ont pas cet effet indésirable. ${ }^{70,77,78}$ Lors d'une vaste $(\mathrm{n}=565)$ analyse rétrospective, Albietz et coll. ont signalé que la régression réfractive était associée à la sécheresse oculaire chronique $\left(\mathrm{p}=0,008\right.$ pour la sécheresse oculaire au $12^{\mathrm{e}}$ mois par rapport à aucune sécheresse oculaire) et à la coloration préexistante de la surface oculaire, mais à un TBUT réduit et à une sensibilité tactile réduite de la cornée ${ }^{81}$ Les auteurs ont fait remarquer que la sécheresse oculaire chez leurs patients décroissait grâce à une prise en charge persistante de la surface oculaire, notamment l'installation d'une occlusion lacrymale (bouchons méatiques), à l'hygiène des paupières, et à l'utilisation constante de gouttes ophtalmiques lubrifiantes. Dans les yeux où l'on pouvait constater une sécheresse oculaire après l'intervention LASIK, l'acuité visuelle non corrigée et les signes et symptômes de sécheresse oculaire se sont améliorés parallèlement ${ }^{81}$, comme il a été déclaré en dehors du contexte clinique. ${ }^{82}$

Dans l'ensemble, ces constatations semblent indiquer que les personnes à risque de sécheresse oculaire après l'intervention LASIK bénéficient de traitements topiques qui rétablissent le film lacrymal et ciblent l'inflammation.

\section{Exacerbation de la sécheresse oculaire après l'intervention LASIK}

Salib et coll. ont mis à l'essai le traitement de la sécheresse oculaire avant l'intervention LASIK au moyen de la cyclosporine par rapport à des larmes artificielles sans agents de conservation chez les patients qui ont des antécédents de sécheresse oculaire $\left(\mathrm{n}=21\right.$ [42 yeux]). ${ }^{83}$ Les patients devant subir une intervention LASIK pour la myopie ont commencé un traitement aux gouttes randomisées deux fois par jour, un mois avant l'intervention. Les deux traitements ont permis d'améliorer la coloration de la surface de la cornée et les symptômes subjectifs de la sécheresse oculaire avant l'intervention. Le traitement topique a été maintenu pendant les trois mois suivant l'intervention. Les symptômes de la sécheresse oculaire se sont aggravés de façon passagère pendant la première semaine d'observation, alors que l'on constatait un rétablissement plus rapide dans le groupe des yeux traités à la cyclosporine par rapport au groupe des yeux traités aux larmes artificielles. De 3 à 12 mois après l'intervention LASIK, les symptômes de la sécheresse oculaire se sont améliorés par rapport à l'évaluation initiale dans les deux groupes de traitement. Les yeux traités à la cyclosporine affichaient une plus grande prévisibilité en réfraction au cours de la période d'observation d'un an et étaient plus susceptibles de se situer à $0,5 \mathrm{D}$ de leur réfraction d'équivalent sphérique ciblée. Cette différence était statistiquement significative au troisième mois $(\mathrm{p}=0,015) .{ }^{83}$ 
Si l'on prévoit utiliser la cyclosporine chez les personnes retenues pour l'intervention LASIK, le traitement devrait commencer un mois ou plus avant l'intervention et se poursuivre pendant trois mois ou plus après l'intervention, en vue d'optimiser les bienfaits lorsque les symptômes de la sécheresse oculaire sont plus incommodants. Le traitement préalable peut permettre aux personnes qui ne seraient pas admissibles autrement à la chirurgie réfractive de subir une intervention LASIK réussie, ${ }^{32}$ et devrait être envisagé pour les personnes qui manifestent une sécheresse oculaire légère ou asymptomatique..$^{66,80}$

En plus du traitement anti-inflammatoire, ${ }^{66,80,83}$ les options thérapeutiques avant et après l'intervention LASIK englobent une vaste gamme d'options exposées dans le guide pratique de $2014,{ }^{8}$ comme les gouttes ophtalmiques lubrifiantes, les lentilles sclérales, l'occlusion lacrymale, la supplémentation en acides gras essentiels, ${ }^{84,85}$ le sérum autologue et la doxycycline par voie orale. Les données périopératoires sur la plupart de ces options sont très limitées. Dans une petite étude portant sur 12 personnes qui présentaient des erreurs réfractives résiduelles après l'intervention LASIK et dont la vision non corrigée s'est améliorée avec l'application de larmes artificielles lubrifiantes, les bouchons méatiques semblaient améliorer considérablement la performance visuelle $(\mathrm{p}<0,0001) .{ }^{86}$ Par ailleurs, Di Pasquale et coll. ont suggéré que les patients qui présentent une instabilité persistante du film lacrymal après le traitement de l'inflammation de la surface oculaire peuvent bénéficier d'un traitement qui consiste à réchauffer l'œil ${ }^{87}$ pour stimuler la sécrétion des glandes de Meibomius et rétablir les lipides des larmes. ${ }^{8,88}$ Dans le cadre d'une étude observationnelle auprès de patients dont la sécheresse oculaire récidivante a persisté pendant un an après l'intervention LASIK, la combinaison de bouchons méatiques, de corticostéroïdes topiques et, s'il y a lieu, de compresses chaudes, a donné lieu à une amélioration subjective des symptômes de la sécheresse oculaire, de même qu'à des augmentations importantes de l'épaisseur de la couche lipidique du film lacrymal et du TBUT. ${ }^{87}$

\section{PRISE EN CHARGE CONCERTÉE DE LA SÉCHERESSE OCULAIRE}

Le tableau 3 contient la liste d'une série d'objectifs pour le traitement préopératoire et postopératoire des patients admissibles à l'intervention de chirurgie oculaire. La liste est générale, en ce sens qu'elle pourrait s'appliquer également à l'opération de cataracte et à la chirurgie réfractive, et potentiellement à d'autres interventions dont les résultats peuvent être compromis par une sécheresse oculaire non maîtrisée. En bref, tous les patients qui sont aiguillés vers une consultation en chirurgie devraient être évalués soigneusement pour déceler les signes et symptômes de maladies de la surface oculaire. Les patients atteints de sécheresse oculaire épisodique ou chronique devraient être traités avant l'intervention chirurgicale dans le but de traiter les signes et les symptômes de la sécheresse oculaire et de stabiliser le film lacrymal et la surface oculaire, pour faire en sorte que les données kératométriques et topographiques soient fiables et précises. Un counseling approprié pendant cette période de traitement est aussi essentiel pour améliorer la compréhension du patient de l'affection et pour gérer les attentes. Après l'intervention, une maladie de la surface oculaire peut se développer ou s'aggraver, au moins temporairement, malgré le recours continu aux traitements de la sécheresse oculaire commencés avant l'intervention chirurgicale. L'objectif des soins pendant cette période devrait être de gérer l'inconfort oculaire et de rétablir une surface oculaire stable et saine.

Tableau 3. Objectifs pour la prise en charge préopératoire et postopératoire de la sécheresse oculaire

\begin{tabular}{|l|l|}
\hline Dans le contexte préopératoire, atteindre: & Dans le contexte postopératoire : \\
\hline 1. Des signes minimaux de sécheresse oculaire à l'examen & $\begin{array}{l}\text { 1. Maintenir un traitement périopératoire si la surface } \\
\text { oculaire est stable }\end{array}$ \\
\hline $\begin{array}{l}\text { 2. Un inconfort minimal et maîtrisé } \\
\text { 3. Un fil lacrymal stable et optimisé }\end{array}$ & $\begin{array}{l}\text { 2. Autrement, intensifier le traitement jusqu'à ce que la } \\
\text { surface oculaire soit stable et qu'elle revienne à un niveau } \\
\text { initial adéquat }\end{array}$ \\
\hline $\begin{array}{l}\text { 4. Des mesures kératométriques stables } \\
\text { (biométrie et topographie) }\end{array}$ & \\
\hline 5. Une réfraction manifeste stable & \\
\hline
\end{tabular}


Si le lieu des soins avant et après l'intervention chirurgicale peut varier quelque peu en fonction des circonstances, en règle générale, les optométristes sont bien placés pour dispenser une grande partie des soins pour la sécheresse oculaire dont ont besoin les patients avant et après l'intervention. La sécheresse oculaire est souvent détectée pour la première fois lorsque les patients reçoivent des soins optométriques. Que le patient soit admissible ou non à l'intervention chirurgicale oculaire, l'optométriste devrait évaluer la surface oculaire et commencer le traitement approprié sans délai. Dans tous les cas, il faut discuter du rôle du film lacrymal et de la surface oculaire avec les candidats à l'intervention chirurgicale pour renforcer la nécessité de bien respecter le traitement et pour éviter la déception après l'opération. Si l'on envisage les lentilles intraoculaires Premium, il faut aviser le patient que ces dispositifs sont particulièrement sensibles aux perturbations de la surface oculaire.

La figure 2 présente un diagramme pour la prise en charge conjointe des patients qui ont besoin d'une intervention chirurgicale oculaire ou qui en font la demande, et qui ont reçu un diagnostic de sécheresse oculaire symptomatique ou asymptomatique. En pareil cas, l'optométriste devrait décrire dans une demande de consultation à l'intention du chirurgien, toutes les constatations concernant la surface oculaire, y compris les rapports subjectifs et les données objectives qui ont mené au diagnostic. Idéalement, l'optométriste prendra en charge l'affection à ce stade précoce, à la fois pour améliorer le confort immédiat du patient et sa fonction visuelle et pour simplifier la prise en charge de l'intervention chirurgicale. La correspondance de l'optométriste devrait décrire les tests effectués et les traitements administrés à ce jour concernant la sécheresse oculaire, de même que les résultats de ces traitements. S'il y a lieu, l'optométriste peut aussi recommander que l'intervention soit retardée pour permettre l'optimisation de la surface oculaire.

Figure 2. Diagramme proposé pour la prise en charge conjointe de la sécheresse oculaire périopératoire

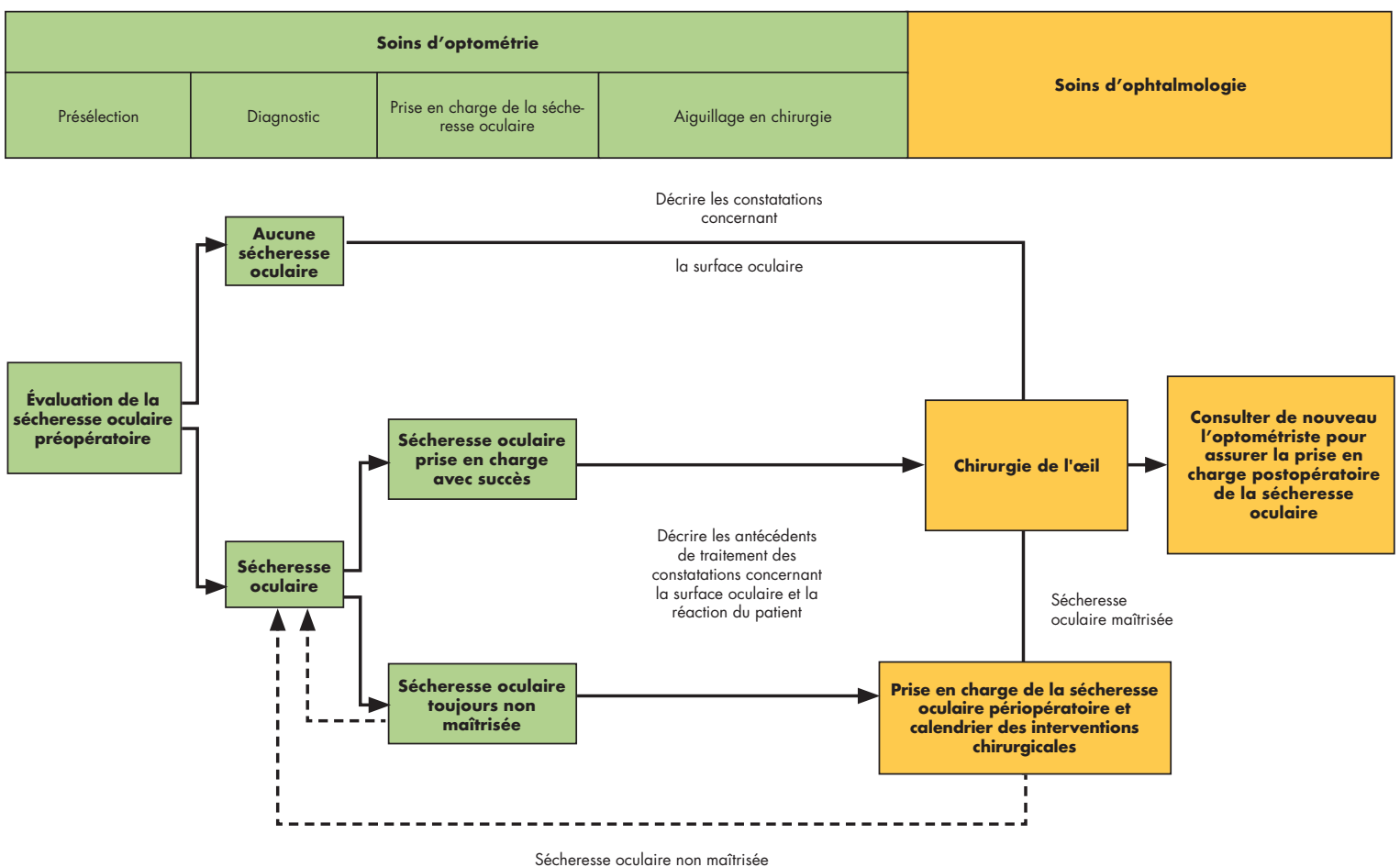

L'ophtalmologiste planifiera l'intervention chirurgicale si la surface oculaire est stable et saine ou il renverra le patient consulter l'optométriste en vue qu'il reçoive un traitement continu ou amélioré contre la sécheresse oculaire. Dans certains cas, l'optométriste peut demander que l'ophtalmologiste se charge des soins au patient, notamment en ce qui concerne la surface oculaire ou la cornée. Après l'intervention, il est généralement approprié que les soins continus pour la sécheresse oculaire soient repris en charge par l'optométriste, à moins que la maladie soit récalcitrante ou que des complications nécessitant une intervention secondaire ou tertiaire ne se manifestent. Dans son 
rapport, le chirurgien devrait décrire la nature et les résultats de l'intervention, de même que tous les changements qui peuvent avoir été faits dans le cadre de la prise en charge postopératoire de la sécheresse oculaire, notamment les changements aux traitements topiques sur ordonnance et en vente libre.

\section{CONCLUSIONS}

La sécheresse oculaire est un trouble inflammatoire chronique que les optométristes et les ophtalmologistes côtoient régulièrement. Elle doit être évaluée et traitée adéquatement chez tous les patients. Les principes du traitement de la sécheresse oculaire sont semblables, que l'on envisage ou non l'intervention chirurgicale oculaire, et devraient suivre les recommandations du document Dépistage, diagnostic et prise en charge de la sécheresse oculaire : guide pratique à l'intention des optométristes canadiens de $2014 .^{8}$ Toutefois, la prise en charge de la sécheresse oculaire est particulièrement importante avant et après certaines interventions chirurgicales, précisément les opérations de chirurgie réfractive et de la cataracte, parce que la sécheresse oculaire non maîtrisée peut placer le patient à risque d'obtenir des résultats chirurgicaux moins qu'optimaux et, à l'inverse, l'intervention chirurgicale provoque ou exacerbe fréquemment la sécheresse oculaire. Par conséquent, il faut retarder l'intervention chirurgicale jusqu'à ce que la surface oculaire se stabilise et qu'un film lacrymal adéquat et approprié soit rétabli, dans toute la mesure du possible. Comme l'illustre la figure 1, le traitement anti-inflammatoire pour les patients atteints de sécheresse oculaire chronique préexistante devrait commencer avant l'intervention chirurgicale et se poursuivre quelques mois après l'intervention. Les recommandations consensuelles pour la prise en charge de la sécheresse oculaire périopératoire figurent au tableau 4.

Tableau 4. Recommandations consensuelles pour la prise en charge de la sécheresse oculaire périopératoire

Recommandation $\mathrm{n}^{\circ} 1$ : Dans le cas des patients chez qui l'on soupçonne la présence d'une douleur neuropathique oculaire, il est important de cerner les affections connexes, comme la douleur neuropathique non oculaire, la dépression, l'anxiété et les troubles du sommeil.

Recommandation $\mathrm{n}^{\circ} 2$ : Selon la fréquence d'utilisation, les formulations sans agents de conservations de produits topiques médicamenteux et non médicamenteux devraient être envisagées pour un traitement avant et après une intervention chirurgicale oculaire.

Recommandation $\mathrm{n}^{\circ} 3$ : Indépendamment de l'inconfort oculaire autodéclaré, les patients qui subissent une opération de la cataracte devraient être évalués pour la détection de signes et symptômes de la sécheresse oculaire.

Recommandation $\mathrm{n}^{\circ} 4$ : La surface oculaire devrait être optimisée avant l'opération de la cataracte, afin d'augmenter l'exactitude et la précision de la biométrie préopératoire et d'améliorer le confort postopératoire et le fonctionnement visuel.

Recommandation $\mathrm{n}^{\circ} 5$ : Les patients affectés d'une sécheresse oculaire symptomatique ou asymptomatique préexistante devraient faire l'objet d'un traitement aux agents anti-inflammatoires avant l'intervention chirurgicale pour prévenir l'exacerbation des symptômes.

Recommandation $n^{\circ} 6$ : Il faut effectuer une évaluation vouée à la sécheresse oculaire dans le cadre du bilan de santé de tous les patients retenus pour l'intervention chirurgicale réfractive.

Recommandation $\mathrm{n}^{\circ} 7$ : Les signes et les symptômes de la sécheresse oculaire chronique, y compris de la sécheresse oculaire légère, devraient être évalués et pris en charge chez tous les candidats à l'intervention chirurgicale oculaire.

Recommandation $\mathrm{n}^{\circ} 8$ : Les patients qui présentent une coloration de la surface oculaire, une instabilité du film lacrymal ou d'autres signes de sécheresse oculaire devraient être informés des risques d'exacerbation de la sécheresse oculaire après l'intervention chirurgicale.

Recommandation $n^{\circ} 9$ : Indépendamment des antécédents de sécheresse oculaire, les patients qui subissent une intervention chirurgicale oculaire devraient être informés que des symptômes de sécheresse oculaire peuvent se manifester après l'intervention.

Pour que les soins périopératoires pour les patients atteints de sécheresse oculaire soient efficients, les optométristes et les ophtalmologistes doivent prendre en charge l'affection conjointement, communiquer entre eux de façon efficace et transmettre des messages uniformes au patient concernant les constatations relatives à la surface oculaire, les risques et la réponse au traitement. Le tableau 1 supplémentaire contient des listes de vérification décrivant les pratiques exemplaires en matière d'échanges entre les chirurgiens et les optométristes. 
Tableau 1 supplémentaire : Renseignements relatifs à la sécheresse oculaire à inclure dans les aiguillages entre les optométristes et les ophtalmologistes

\begin{tabular}{|c|c|c|}
\hline & Optometrist to ophthalmologist & Ophthalmologist back to optometrist \\
\hline 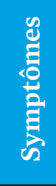 & $\left.\begin{array}{l}\text { - Sensation } \\
\text { - Vision }\end{array}\right\} \begin{array}{l}\text { Préciser } \\
\text { le calendrier }\end{array}$ & $\begin{array}{l}\text { - Sensation } \\
\text { - Vision } \\
\text { - Description d'intervention chirurgicale récente } \\
\text { - Résultats (inclure la réfraction postopératoire si elle } \\
\text { a été effectuée) }\end{array}$ \\
\hline 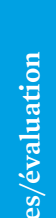 & \multicolumn{2}{|c|}{$\begin{array}{l}\text { Constatations } \\
\text { - Score symptomatique (questionnaire) } \\
\text { - } \text { Stabilité lacrymale (NIBUT/TBUT) } \\
\text { - Fonctionnement des glandes de Meibomius } \\
\text { - } \text { Autres tests (p. ex., osmolarité du film lacrymal) }\end{array}$} \\
\hline क्ष & \multicolumn{2}{|c|}{$\begin{array}{l}\text { Description de la sécheresse oculaire/surface oculaire } \\
\text { - Gravité } \\
\text { - Actuellement stable? (si oui, depuis combien de temps a-t-on obtenu la stabilité?) }\end{array}$} \\
\hline 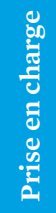 & $\begin{array}{l}\text { - Traitements actuels et antérieurs } \\
\text { - Problèmes de tolérance, s'il y a lieu } \\
\text { - Antécédents d'adhésion }\end{array}$ & $\begin{array}{l}\text { Modifications postopératoires apportées à la prise } \\
\text { en charge de la surface oculaire, y compris les produits sans } \\
\text { ordonnance? (Si la réponse est oui, veuillez préciser les } \\
\text { produits et le dosage) }\end{array}$ \\
\hline 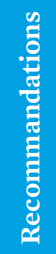 & $\begin{array}{l}\text { Le patient devrait-il subir une intervention } \\
\text { chirurgicale le plus tôt possible? } \\
\text { - Si la réponse est non, quelle étape de } \\
\text { traitement doit être atteinte avant de } \\
\text { planifier l'intervention chirurgicale? }\end{array}$ & $\begin{array}{l}\text { - Instructions pour les soins postopératoires } \\
\text { - Prochaine visite prévue à la clinique ou retour aux soins } \\
\text { de l'optométriste ou prise en charge conjointe } \\
\text { - Objectifs du patient concernant sa vision }\end{array}$ \\
\hline
\end{tabular}

\section{REMERCIEMENTS}

Ces travaux ont été financés par une subvention sans restriction provenant d’Allergan Inc.

DIVULGATIONS

J. Ashkenas a reçu un soutien financier d'Allergan Canada par le biais de SCRIPT (Toronto, Canada) pour sa participation au projet.

Au cours des trois dernières années, E. Bitton a reçu des honoraires et/ou du financement des sociétés suivantes : Akorn, ALCON, Allergan, American Academy of Optometry, Association canadienne des optométristes, COETF, CooperVision, Labtician, I-Med Pharma Inc., Jobson Publishing, McCann Medical, Optician Journal, Novartis, Orimed, Santen, Shire, et TBWA World Health.

B. Caffery a reçu des honoraires d'experts-conseils de Santen, de Shire, d'Allergan, de Novartis, d'Alcon et de Labtician au cours des trois dernières années.

J.-S. Dufour a reçu des honoraires d'Allergan, de Novartis et de Shire au cours des trois dernières années.

P. M. Karpecki a reçu des honoraires d'experts-conseils des sociétés suivantes : Akorn, AMO/JJV, Alcon, Allergan, B+L, Blephex, BVI, BioTissue, Bruder Healthcare, Eyegate, Focus Labs, Oculus, OcuSoft, Shire, Rendia, TearLab, TearScience, et Zeiss.

L. Michaud a reçu des honoraires et/ou du financement des sociétés suivantes : Alcon, Allergan, COETF, Cooper Vision, Johnson \& Johnson Vision Care, Valeant, Blanchard Labs, Genzyme, Shire, Knights Ophthalmics, et Santen, au cours des trois dernières années.

P. Neumann a reçu du financement d'Allergan Canada pour des services de conférencier et d'experts-conseils au cours des trois dernières années.

V. Pegado n'a pas de relation de financement à divulguer. • 
L. Racine a reçu des honoraires d'experts-conseils d'Allergan, de Bausch and Lomb, de Johnson and Johnson Vision, de Shire, de Santen et de Valeant.

A. Webber a reçu des honoraires de conseiller et de conférencier d'Allergan, de Bausch and Lomb et de Shire, au cours des trois dernières années.

\section{RÉFÉRENCES}

1. Smith JA, Albeitz J, Begley C et al. The Epidemiology of Dry Eye Disease: Report of the Epidemiology Subcommittee of the International Dry Eye WorkShop (2007). Ocul Surf 2007; 5(2): 93-107.

2. Moss SE, Klein R, Klein BE. Incidence of dry eye in an older population. Arch Ophthalmol 2004; 122(3): 369-73.

3. Sagdik HM, Ugurbas SH, Can M et al. Tear film osmolarity in patients with diabetes mellitus. Ophthalmic Res 2013; 50(1): 1-5.

4. Begley CG, Caffery B, Nichols KK, Chalmers R. Responses of contact lens wearers to a dry eye survey. Optom Vis Sci 2000; 77(1): 40-6.

5. Schaumberg DA, Sullivan DA, Dana MR. Epidemiology of dry eye syndrome. Adv Exp Med Biol 2002; 506(Pt B): 989-98.

6. Vivino FB, Carsons SE, Foulks G et al. New treatment guidelines for Sjogren's Disease. Rheum Dis Clin North Am 2016; 42(3): 531-51.

7. Lekskul M, Fracht HU, Cohen EJ et al. Nontraumatic corneal perforation. Cornea 2000; 19: 3-319.

8. Prokopich C, Bitton E, Caffery B et al. Screening, Diagnosis and Management of Dry Eye Disease: Practical Guidelines for Canadian Optometrists. Can J Optometry 2014; 76 (Suppl 1): 1-31.

9. Aragona P, Aguennouz M, Rania L et al. Matrix metalloproteinase 9 and transglutaminase 2 expression at the ocular surface in patients with different forms of dry eye disease. Ophthalmology 2015; 122(1): 62-71.

10. Bron AJ, Tomlinson A, Foulks GN et al. Rethinking dry eye disease: a perspective on clinical implications. Ocul Surf 2014; 12(2 Suppl): S1-31.

11. Foster CS. Dry Eye Syndrome (Keratoconjunctivitis Sicca) Medication 2016. http://emedicine.staging.medscape.com/article/1210417medication. Accessed January 2017.

12. Holland EJ, Luchs J, Karpecki PM et al. Lifitegrast for the treatment of dry eye disease: Results of a phase iii, randomized, double-masked, placebo-controlled trial (OPUS-3). Ophthalmology 2017; 124(1): 53-60.

13. Sheppard JD, Torkildsen GL, Lonsdale JD et al. Lifitegrast ophthalmic solution $5.0 \%$ for treatment of dry eye disease: results of the OPUS-1 phase 3 study. Ophthalmology 2014; 121(2): 475-83.

14. Tauber J, Karpecki P, Latkany R et al. Lifitegrast ophthalmic solution $5.0 \%$ versus placebo for treatment of dry eye disease: Results of the randomized phase III OPUS-2 study. Ophthalmology 2015; 122(12): 2423-31.

15. Baudouin C, Figueiredo FC, Messmer EM et al. A randomized study of the efficacy and safety of $0.1 \%$ cyclosporine A cationic emulsion in treatment of moderate to severe dry eye. Eur J Ophthalmol 2017; 27(5): 520-30.

16. Leonardi A, Van Setten G, Amrane M et al. Efficacy and safety of $0.1 \%$ cyclosporine A cationic emulsion in the treatment of severe dry eye disease: a multicenter randomized trial. Eur J Ophthalmol 2016; 26(4): 287-96.

17. Robert PY, Cochener B, Amrane M et al. Efficacy and safety of a cationic emulsion in the treatment of moderate to severe dry eye disease: a randomized controlled study. Eur J Ophthalmol 2016; 26(6): 546-55

18. Cetinkaya S, Mestan E, Acir NO et al. The course of dry eye after phacoemulsification surgery. BMC Ophthalmol 2015; 15: 68.

19. Cho YK, Kim MS. Dry eye after cataract surgery and associated intraoperative risk factors. Korean J Ophthalmol 2009; 23(2): 65-73.

20. Kasetsuwan N, Satitpitakul V, Changul T, Jariyakosol S. Incidence and pattern of dry eye after cataract surgery. PLoS One 2013; 8(11): e78657.

21. Turu L, Alexandrescu C, Stana D, Tudosescu R. Dry eye disease after LASIK. J Med Life 2012; 5(1): 82-4.

22. McMonnies CW. The potential role of neuropathic mechanisms in dry eye syndromes. J Optom 2017; 10(1): 5-13,

23. Belmonte C, Nichols JJ, Cox SM et al. TFOS DEWS II pain and sensation report. Ocul Surf 2017; 15(3): 404-37.
24. Rosenthal P, Borsook D. Ocular neuropathic pain. Br J Ophthalmol 2016; 100(1): 128-34.

25. Trattler W, Reilly C, Goldberg D et al. Cataract and Dry Eye: Prospective Health Assessment of Cataract Patients Ocular Surface Study. Proceedings of the American Society of Cataract and Refractive Surgery; San Diego, CA; 2011.

26. Arciniega JC, Wojtowicz JC, Mohamed EM, McCulley JP. Changes in the evaporation rate of tear film after digital expression of Meibomian glands in patients with and without dry eye. Cornea 2011; 30(8): 843-7.

27. Lemp MA, Baudouin C, Baum J et al. The Definition and Classification of Dry Eye Disease: Report of the Definition and Classification Subcommittee of the International Dry Eye WorkShop (2007). Ocul Surf 2007; 5(2): 75-92.

28. Denoyer A, Rabut G, Baudouin C. Tear film aberration dynamics and vision-related quality of life in patients with dry eye disease. Ophthalmology 2012; 119(9): 1811-8.

29. Koh S. Mechanisms of visual disturbance in dry eye. Cornea 2016; 35 Suppl 1: S83-8.

30. Koh S, Maeda N, Hirohara Y et al. Serial measurements of higher-order aberrations after blinking in patients with dry eye. Invest Ophthalmol Vis Sci 2008; 49(1): 133-8.

31. Montes-Mico R, Caliz A, Alio JL. Changes in ocular aberrations after instillation of artificial tears in dry-eye patients. J Cataract Refract Surg 2004; 30(8): 1649-52.

32. Ambrosio R, Jr., Tervo T, Wilson SE. LASIK-associated dry eye and neurotrophic epitheliopathy: pathophysiology and strategies for prevention and treatment. J Refract Surg 2008; 24(4): 396-407.

33. Chao C, Golebiowski B, Stapleton F. The role of corneal innervation in LASIK-induced neuropathic dry eye. Ocul Surf 2014; 12(1): 32-45.

34. Nettune GR, Pflugfelder SC. Post-LASIK tear dysfunction and dysesthesia. Ocul Surf 2010; 8(3): 135-45.

35. Liu H, Thibos L, Begley CG, Bradley A. Measurement of the time course of optical quality and visual deterioration during tear breakup. Invest Ophthalmol Vis Sci 2010; 51(6): 3318-26.

36. van Landingham SW, West SK, Akpek EK et al. Impact of dry eye on reading in a population-based sample of the elderly: the Salisbury Eye Evaluation. Br J Ophthalmol 2014; 98(5): 639-44.

37. Deschamps N, Ricaud X, Rabut $\mathrm{G}$ et al. The impact of dry eye disease on visual performance while driving. Am J Ophthalmol 2013; 156(1): 184-9 el83.

38. De Paiva CS, Harris LD, Pflugfelder SC. Keratoconus-like topographic changes in keratoconjunctivitis sicca. Cornea 2003; 22(1): $22-4$

39. Epitropoulos AT, Matossian C, Berdy GJ et al. Effect of tear osmolarity on repeatability of keratometry for cataract surgery planning. $\mathrm{J}$ Cataract Refract Surg 2015; 41(8): 1672-7.

40. Kohlhaas M. Corneal sensation after cataract and refractive surgery. J Cataract Refract Surg 1998; 24(10): 1399-409.

41. Nariani A, Gupta P. Dry eye and refractive surgery outcomes. Curr Ophthalmol Rep 2016; 4: 8-14.

42. Yokoi N, Uchino M, Uchino $\mathrm{Y}$ et al. Importance of tear film instability in dry eye disease in office workers using visual display terminals: the Osaka study. Am J Ophthalmol 2015; 159(4): 748-54

43. Gupta P. Cataract surgery in patients with Meibomian gland dysfunction. Cataract Refract Surg Today 2015: 77-8.

44. Potvin R. Cataracts in Canada: Introduction. Can J Optometry 2015; 77 (Suppl. 1): 4-6.

45. Canadian Association of Optometrists. Cataracts surgery in Canada: What you need to know according to the Canadian Journal of Optometry 2015. https://opto.ca/cataracts-surgery-in-canada-what-youneed-to-know-according-to-the-canadian-journal-of-optometry. 
Accessed January 2017.

46. Hatch WV, Campbell Ede L, Bell CM et al. Projecting the growth of cataract surgery during the next 25 years. Arch Ophthalmol 2012 130(11): 1479-81.

47. Donnenfeld ED, Solomon R, Roberts CW et al. Cyclosporine 0.05\% to improve visual outcomes after multifocal intraocular lens implantation. J Cataract Refract Surg 2010; 36(7): 1095-100.

48. Solomon R, Donnenfeld ED. Refractive Intraocular Lenses: Multifocal and Phakic IOLs. Int Ophthalmol Clin 2006; 46: 123-43.

49. Kuroda T, Fujikado T, Maeda N et al. Wavefront analysis of higher-order aberrations in patients with cataract. J Cataract Refract Surg 2002; 28(3): 438-44.

50. Rocha KM, Nose W, Bottos K et al. Higher-order aberrations of age-related cataract. J Cataract Refract Surg 2007; 33(8): 1442-6.

51. Zetterberg M, Celojevic D. Gender and cataract--the role of estrogen. Curr Eye Res 2015; 40(2): 176-90.

52. Johnston J. The cataract patient is a dry eye patient. Rev Cornea Contact Lenses 2015. http://connection.ebscohost.com/c/articles/112930642/cataract-patient-dry-eye-patient. Accessed January 2017.

53. Bron AJ, Abelson MB, Ousler G et al. Methodologies to diagnose and monitor dry eye disease: Report of the Diagnostic Methodology Subcommittee of the International Dry Eye WorkShop. Ocul Surf 2007; 5(2): 108-52.

54. Kim P, Plugfelder S, Slomovic AR. Top 5 pearls to consider when implanting advanced-technology IOLs in patients with ocular surface disease. Int Ophthalmol Clin 2012; 52(2): 51-8.

55. Li XM, Hu L, Hu J, Wang W. Investigation of dry eye disease and analysis of the pathogenic factors in patients after cataract surgery. Cornea 2007; 26(9 Suppl 1): S16-20.

56. Jiang D, Xiao X, Fu T et al. Transient tear film dysfunction after cataract surgery in diabetic patients. PLoS One 2016; 11(1): e0146752.

57. Mencucci R, Boccalini C, Caputo R, Favuzza E. Effect of a hyaluronic acid and carboxymethylcellulose ophthalmic solution on ocular comfort and tear-film instability after cataract surgery. J Cataract Refract Surg 2015; 41(8): 1699-704.

58. Sanchez MA, Arriola-Villalobos P, Torralbo-Jimenez P et al. The effect of preservative-free HP-Guar on dry eye after phacoemulsification: a flow cytometric study. Eye (Lond) 2010; 24(8): $1331-7$.

59. Jee D, Park M, Lee HJ et al. Comparison of treatment with preservative-free versus preserved sodium hyaluronate $0.1 \%$ and fluorometholone $0.1 \%$ eyedrops after cataract surgery in patients with preexisting dry-eye syndrome. J Cataract Refract Surg 2015 41(4): 756-63.

60. Chen M, Gong L, Sun X et al. A comparison of cyclosporine $0.05 \%$ ophthalmic emulsion versus vehicle in Chinese patients with moderate to severe dry eye disease: an eight-week, multicenter, randomized, double-blind, parallel-group trial. J Ocul Pharmacol Ther 2010; 26(4): 361-6.

61. Rao SN. Topical cyclosporine $0.05 \%$ for the prevention of dry eye disease progression. J Ocul Pharmacol Ther 2010; 26(2): 157-64.

62. Rao SN. Reversibility of dry eye deceleration after topical cyclosporine $0.05 \%$ withdrawal. J Ocul Pharmacol Ther 2011; 27(6): 603-9.

63. Chung YW, Oh TH, Chung SK. The effect of topical cyclosporine $0.05 \%$ on dry eye after cataract surgery. Korean J Ophthalmol 2013 27(3): 167-71.

64. Lee JH, Song IS, Kim KL, Yoon SY. Effectiveness and optical quality of topical 3.0\% Diquafosol versus $0.05 \%$ Cyclosporine A in dry eye patients following cataract surgery. J Ophthalmol 2016; 8150757.

65. Hamada S, Moore TC, Moore JE et al. Assessment of the effect of cyclosporine-A $0.05 \%$ emulsion on the ocular surface and corneal sensation following cataract surgery. Cont Lens Anterior Eye 2016; 39(1): 15-9.

66. Ursea R, Purcell TL, Tan BU et al. The effect of cyclosporine A (Restasis) on recovery of visual acuity following LASIK. J Refract Surg 2008; 24(5): 473-6.

67. McGhee CN, Orr D, Kidd B et al. Psychological aspects of excimer laser surgery for myopia: reasons for seeking treatment and patient satisfaction. Br J Ophthalmol 1996; 80(10): 874-9.

68. Toda I, Asano-Kato N, Hori-Komai Y, Tsubota K. Ocular surface treatment before laser in situ keratomileusis in patients with severe dry eye. J Refract Surg 2004; 20(3): 270-5.

69. Xie W. Recent advances in laser in situ keratomileusis-associated dry eye. Clin Exp Optom 2016; 99(2): 107-12.

70. Albietz JM, Lenton LM. Management of the ocular surface and tear film before, during, and after laser in situ keratomileusis. J Refract Surg 2004; 20(1): 62-71.

71. Torricelli AA, Bechara SJ, Wilson SE. Screening of refractive surgery candidates for LASIK and PRK. Cornea 2014; 33(10): 1051-5.

72. Garcia-Zalisnak D, Nash D, Yeu E. Ocular surface diseases and corneal refractive surgery. Curr Opin Ophthalmol 2014; 25(4): 264-9.

73. Torricelli AA, Santhiago MR, Wilson SE. Topical cyclosporine a treatment in corneal refractive surgery and patients with dry eye. $J$ Refract Surg 2014; 30(8): 558-64.

74. Denoyer A, Landman E, Trinh L et al. Dry eye disease after refractive surgery: comparative outcomes of small incision lenticule extraction versus LASIK. Ophthalmology 2015; 122(4): 669-76.

75. Wang B, Naidu RK, Chu R et al. Dry Eye Disease following refractive surgery: A 12-month follow-up of SMILE versus FS-LASIK in high myopia. J Ophthalmol 2015; 132417.

76. Bower KS, Sia RK, Ryan DS et al. Chronic dry eye in photorefractive keratectomy and laser in situ keratomileusis: Manifestations, incidence, and predictive factors. J Cataract Refract Surg 2015; 41(12): 2624-34.

77. Albietz JM, Lenton LM, McLennan SG. Effect of laser in situ keratomileusis for hyperopia on tear film and ocular surface. J Refract Surg 2002; 18(2): 113-23.

78. Esquenazi S. Five-year follow-up of laser in situ keratomileusis for hyperopia using the Technolas Keracor $117 \mathrm{C}$ excimer laser. J Refract Surg 2004; 20(4): 356-63.

79. Goto T, Zheng X, Klyce SD et al. Evaluation of the tear film stability after laser in situ keratomileusis using the tear film stability analysis system. Am J Ophthalmol 2004; 137(1): 116-20.

80. Peyman GA, Sanders DR, Batlle JF et al. Cyclosporine $0.05 \%$ ophthalmic preparation to aid recovery from loss of corneal sensitivity after LASIK. J Refract Surg 2008; 24(4): 337-43.

81. Albietz JM, Lenton LM, McLennan SG. Chronic dry eye and regression after laser in situ keratomileusis for myopia. J Cataract Refract Surg 2004; 30(3): 675-84.

82. Sall K, Stevenson OD, Mundorf TK, Reis BL. Two multicenter, randomized studies of the efficacy and safety of cyclosporine ophthalmic emulsion in moderate to severe dry eye disease. CsA Phase 3 Study Group. Ophthalmology 2000; 107(4): 631-9.

83. Salib GM, McDonald MB, Smolek M. Safety and efficacy of cyclosporine $0.05 \%$ drops versus unpreserved artificial tears in dry-eye patients having laser in situ keratomileusis. J Cataract Refract Surg 2006; 32(5): 772-8

84. Sheppard JD, Jr., Singh R, McClellan AJ et al. Long-term supplementation with n-6 and n-3 PUFAs improves moderate-to-severe keratoconjunctivitis sicca: A randomized double-blind clinical trial. Cornea 2013; 32(10): 1297-304.

85. Zhu W, Wu Y, Li G et al. Efficacy of polyunsaturated fatty acids for dry eye syndrome: a meta-analysis of randomized controlled trials. Nutr Rev 2014; 72(10): 662-71.

86. Khalil MB, Latkany RA, Speaker MG, Yu G. Effect of punctal plugs in patients with low refractive errors considering refractive surgery. J Refract Surg 2007; 23(5): 467-71.

87. Di Pascuale MA, Liu TS, Trattler W, Tseng SC. Lipid tear deficiency in persistent dry eye after laser in situ keratomileusis and treatment results of new eye-warming device. J Cataract Refract Surg 2005; 31(9): 1741-9.

88. Schaumberg DA, Nichols JJ, Papas EB et al. The international workshop on Meibomian gland dysfunction: report of the subcommittee on the epidemiology of, and associated risk factors for, MGD. Invest Ophthalmol Vis Sci 2011; 52(4): 1994-2005.

89. Abelson M, Rosenthal P, McLaughlin J. Neuropathic pain: The artifice of dry eye. Review of Ophthalmology 2016; 23(1): 1-3.

90. Belmonte C, Acosta MC, Merayo-Lloves J, Gallar J. What causes eye pain? Curr Ophthalmol Rep 2015; 3(2): 111-21.

91. Galor A, Batawi H, Felix ER et al. Incomplete response to artificial tears is associated with features of neuropathic ocular pain. $\mathrm{Br} \mathrm{J}$ Ophthalmol 2016; 100(6): 745-9.

92. Crane AM, Levitt RC, Felix ER et al. Patients with more severe symptoms of neuropathic ocular pain report more frequent and severe chronic overlapping pain conditions and psychiatric disease. Br J Ophthalmol 2017; 101(2): 227-31 
93. Shtein RM, Harper DE, Pallazola V et al. Discordant dry eye disease (An American Ophthalmological Society Thesis). Trans Am Ophthalmol Soc 2016; 114: T4.

94. Baudouin C. Side effects of antiglaucomatous drugs on the ocular surface. Curr Opin Ophthalmol 1996; 7(2): 80-6.

95. Baudouin C, Renard JP, Nordmann JP et al. Prevalence and risk factors for ocular surface disease among patients treated over the long term for glaucoma or ocular hypertension. Eur J Ophthalmol 2012: 0.

96. Conlon R, Saheb H, Ahmed, II. Glaucoma treatment trends: a review. Can J Ophthalmol 2017; 52(1): 114-24.

97. Pisella PJ, Pouliquen P, Baudouin C. Prevalence of ocular symptoms and signs with preserved and preservative free glaucoma medication. Br J Ophthalmol 2002; 86(4): 418-23.

98. Garcia-Feijoo J, Sampaolesi JR. A multicenter evaluation of ocular surface disease prevalence in patients with glaucoma. Clin Ophthalmol 2012; 6: 441-6.

99. Leung EW, Medeiros FA, Weinreb RN. Prevalence of ocular surface disease in glaucoma patients. J Glaucoma 2008; 17(5): 350-5.

100. Ramli N, Supramaniam G, Samsudin A et al. Ocular surface disease in glaucoma: Effect of polypharmacy and preservatives. Optom Vis Sci 2015; 92(9): e222-6.

101. Warcoin E, Clouzeau C, Roubeix C et al. Hyperosmolarity and benzalkonium chloride differently stimulate inflammatory markers in conjunctiva-derived epithelial cells in vitro. Ophthalmic Res 2017; 58(1): 40-8.

102. Broadway DC, Grierson I, Sturmer J, Hitchings RA. Reversal of topical antiglaucoma medication effects on the conjunctiva. Arch Ophthalmol 1996; 114(3): 262-7.

103. Kersey JP, Broadway DC. Corticosteroid-induced glaucoma: a review of the literature. Eye (Lond) 2006; 20(4): 407-16.

104. Skalicky SE, Goldberg I, McCluskey P. Ocular surface disease and quality of life in patients with glaucoma. Am J Ophthalmol 2012; 153(1): 1-9 e2.

105. Fechtner RD, Godfrey DG, Budenz D et al. Prevalence of ocular surface complaints in patients with glaucoma using topical intraocular pressure-lowering medications. Cornea 2010; 29(6): 618-21.

106. Saini M, Dhiman R, Dada T et al. Topical cyclosporine to control ocular surface disease in patients with chronic glaucoma after longterm usage of topical ocular hypotensive medications. Eye (Lond) 2015; 29(6): 808-14.

107. Batra R, Tailor R, Mohamed S. Ocular surface disease exacerbated glaucoma: optimizing the ocular surface improves intraocular pressure control. J Glaucoma 2014; 23(1): 56-60.

108. de Jong C, Stolwijk T, Kuppens E et al. Topical timolol with and without benzalkonium chloride: epithelial permeability and autofluorescence of the cornea in glaucoma. Graefes Arch Clin Exp Ophthalmol 1994; 232(4): 221-4.

109. Gayton JL, Van Der Karr M, Sanders V. Combined cataract and glaucoma surgery: trabeculectomy versus endoscopic laser cycloablation. J Cataract Refract Surg 1999; 25(9): 1214-9.

110. Tanbakouee E, Ghoreishi M, Aghazadeh-Amiri M et al. Photore- fractive keratectomy for patients with preoperative low Schirmer test value. J Curr Ophthalmol 2016; 28(4): 176-80.

111. Al-Swailem SA. Graft failure: II. Ocular surface complications. Int Ophthalmol 2008; 28(3): 175-89.

112. Sheppard JJ. Prevalence of dry eye in planned penetrating or endothelial keratoplasty. abstractsnet.com/handouts/0225_Dry_eye_ prevalence_WCC_2015.pptx. Accessed January 2017.

113. Tan DT, Dart JK, Holland EJ, Kinoshita S. Corneal transplantation. Lancet 2012; 379(9827): 1749-61.

114. Shousha MA, Yoo SH, Kymionis GD et al. Long-term results of femtosecond laser-assisted sutureless anterior lamellar keratoplasty. Ophthalmology 2011; 118(2): 315-23.

115. Li M, Zhang M, Lin Y et al. Tear function and goblet cell density after pterygium excision. Eye (Lond) 2007; 21: 224-8.

116. Chhadva P, Alexander A, McClellan AL et al. The impact of conjunctivochalasis on dry eye symptoms and signs. Invest Ophthalmol Vis Sci 2015; 56(5): 2867-71.

117. Di Pascuale MA, Espana EM, Kawakita T, Tseng SC. Clinical characteristics of conjunctivochalasis with or without aqueous tear deficiency. Br J Ophthalmol 2004; 88(3): 388-92.

118. Hara S, Kojima T, Ishida R et al. Evaluation of tear stability after surgery for conjunctivochalasis. Optom Vis Sci 2011; 88(9): 1112-8.

119. Acera A, Vecino E, Duran JA. Tear MMP-9 levels as a marker of ocular surface inflammation in conjunctivochalasis. Invest Ophthalmol Vis Sci 2013; 54(13): 8285-91.

120. Yu EY, Leung A, Rao S, Lam DS. Effect of laser in situ keratomileusis on tear stability. Ophthalmology 2000; 107(12): 2131-5.

121. Saedon H, Nosek J, Phillips J et al. Ocular surface effects of repeated application of povidone iodine in patients receiving frequent intravitreal injections. Cutan Ocul Toxicol 2017: 1-4.

122. Bagheri A, Najmi H, Salim RE, Yazdani S. Tear condition following unilateral ptosis surgery. Orbit 2015; 34(2): 66-71.

123. Prischmann J, Sufyan A, Ting JY et al. Dry eye symptoms and chemosis following blepharoplasty: a 10-year retrospective review of 892 cases in a single-surgeon series. JAMA Facial Plast Surg 2013; 15(1): 39-46.

124. Saadat D, Dresner SC. Safety of blepharoplasty in patients with preoperative dry eyes. Arch Facial Plast Surg 2004; 6(2): 101-4.

125. Lee SY, Wong TT, Chua J et al. Effect of chronic anti-glaucoma medications and trabeculectomy on tear osmolarity. Eye (Lond) 2013; 27(10): 1142-50.

126. Boimer C, Birt CM. Preservative exposure and surgical outcomes in glaucoma patients: The PESO study. J Glaucoma 2013; 22(9): 730-5.

127. Li Q, Fu T, Yang $\mathrm{J}$ et al. Ocular surface changes after strabismus surgery with different incisions. Graefes Arch Clin Exp Ophthalmol 2015 ; $253(3): 431-8$. 